\title{
A MOBILE FULL-TIME DAILY SYSTEM FOR FETAL MONITORING
}

\author{
Artem Bureev ${ }^{1}$, Elena Vaganova $^{2}$, Dmitry $_{\text {Zhdanov }}{ }^{1,2^{*}}$, Ivan Zemlyakov ${ }^{1}$, \\ Ekaterina Dikman ${ }^{1}$ \\ ${ }^{1}$ Diagnostika + LLC , 634055, Tomsk, Russia \\ ${ }^{2}$ National Research Tomsk State University, 634050, Tomsk, Russia
}

\begin{abstract}
The article describes a mobile hardware and software system designed for daily monitoring of the state of fetal and maternal cardiovascular systems. The assessment is carried out by means of recording and further online analysis of acoustic data, obtained from the abdominal surface of a pregnant woman's body. The components and operating principles of the hardware and software system designed are described. The results of experimental studies aimed at assessing the applicability of a method of acoustic data analysis implemented in the system developed are shown. The results obtained have been compared with the results obtained using cardiotocography.
\end{abstract}

\section{Introduction}

Fetal monitoring of the state of the cardiovascular system in the antenatal period is the most common non-invasive method of a fetus wellbeing control. The relevance of the method is confirmed by numerous publications (both professional and popular scientific) in Russian and foreign medical literature. Currently used methods are based on the analysis of trends in fetal heart rate, resulting in the construction of a rhythmogram of between systolic time intervals, known as a cardiotocogram. The procedure for its construction is called cardiotocography. Currently, this technique is based on the ultrasound examination for outpatients, where physical heart contractions of a fetus are assessed with a Doppler sonography. Frequent use of ultrasound in medicine is not considered to be fully secure. Until now there is no definite answer whether the ultrasound acoustic power (ALARA) within 30-40 minutes is safe for fetal development $[1,2]$. There is very little information on possible hidden biological effects of ultrasound on the development of a human embryo or fetus. There is evidence that even low power ultrasound can affect the development of brains and visceral organs of small animals. However, direct adaptation of these results to human beings appears invalid [1,2].

In Russia according to the federal standard of care for women with normal pregnancy (Order of the Ministry of Health of the Russian Federation, Sept. 14, 2006 № 662) the number of recommended sessions of cardiotocography should not exceed three times. Some researchers still believe that the level of ultrasound power during cardiotocography is quite

* Corresponding author: dim@diagnostic.tom.ru 
low and the technique is not contraindicated for pregnant women, even in case of five or more sessions [1]. However the predictive value of cardiotocogram does not surpass two weeks. Thus long-term monitoring in the case of a pathology of fetal development may need to involve additional safer methods.

At the present time, cardiotocograms obtained by means of complex electrodes applied to the anterior abdominal wall of the pregnant woman, are increasingly frequently used as an alternative to ultrasonic methods.

The procedure for obtaining cardiotocograms is usually performed in a hospital, under the condition of a complete physical rest of a pregnant. This is due to the fact that the amplitude of the cardiotocogram teeth, recorded from the abdominal wall of a mother, is about 100 times smaller than the amplitude of its electrocardiogram. Nevertheless there are many cases when portable devices for fetal daily monitoring are necessary.

Monica Healthcare (GB) has developed a wearable device for daily monitoring of fetal heart rate Monica AN24. The device measures the fetal and maternal heart rate, and other parameters by recording fetal and maternal electrocardiograms as well as electromyograms of maternal uterine muscles. The device is a hardware system consisting of a portable battery-powered recorder (about 350 gr.), and the doctor's computer work station on the basis of a personal computer with specialized software. It practically helps doctors to avoid false decisions [3]. However despite the possibility of the continuous monitoring, reliability and safety of the method, there is a certain complexity of fetal electrocardiogram recording and fetal heart rate assessment. Receiving of high quality cardiotocograms using MONICA AN24 is only possible with limited mobility of the patient (even better in the supine position) $[3,4]$.

Certainly fetal electrocardiogram has undeniable advantages. However, separating the basic QRS-complex (or at least R-wave fetal electrocardiogram) against the background electromiotic activity skeletal and respiratory muscles, as well as the maternal electrocardiogram is challenging and requires significant computing resources. It makes difficult to assess fetal heart rates in real time. In addition, for improvement of the signal / noise ratio patient's preparation for the electrode application requires careful processing of the skin surface of her skin with a special fluid, removing the horny layer of the epidermis [3].

\section{Methods}

The team of project developers have proposed a portable fetal monitor using passive acoustic detection of fetal heart tones recorded (phonocardiography) [5]. Currently there are no commercially available or being under research and development wearable daily monitors for control of fetal and maternal heart rates using phonocardiography. Various device for home use, manufactured in China and other countries of Southeast Asia and allow to listen to the fetal heart tones. However due to due to external factors they can not be assigned to medical daily monitors.

The developed prototype of a mobile daily monitor of fetal and maternal heart rates consists of four units (Figure 1): 


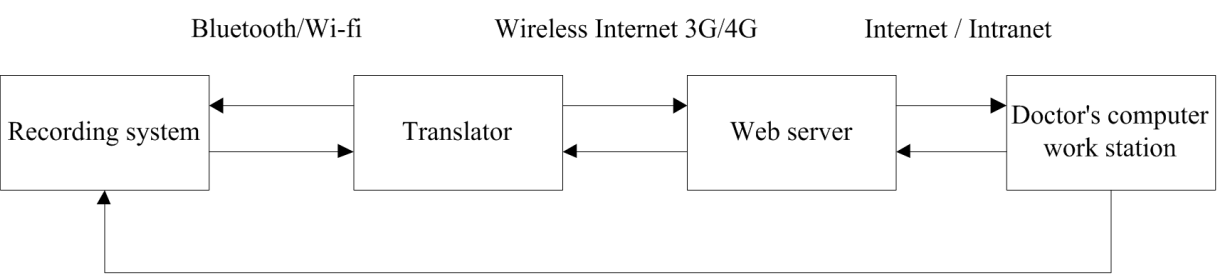

Figure 1. Block scheme of a prototype of a mobile daily monitor.

A wearable recorder is a compact battery-powered device $(95 \times 115 \times 30 \mathrm{~mm})$. Fetal heart tones are recorded from the anterior abdominal wall of a pregnant woman by piezoceramic transducers adjusted by a doctor. Their location depends on the fetal attitude (Figure 2).

In the developed mobile version of the system a smart/mobile phone (with Internet access), is a transitional translator. Special software receives and processed signals and sends them to a remote server via wireless Internet. Before sending the data they are encoded and archived in accordance with the Federal Law of the Russian Federation regarding personal data protection (№153, 27.07.2006). Ddepersonalization of data transmissed is performed by a system of personal codes and markers. The experimental results showed that the computing capacity of a Android 4.x smartphone of a low price range (less than $\$ 50$ ) is enough for the task.

Besides reception, processing and transmission of data, a smartphone allows to get information about the recording system. The processed signals are presented in text and graphic form. The mode of voice and text communication with your doctor is available. In case of emergency signals from the recording system are transmitted to the doctor's computer work station through a translator and server online.

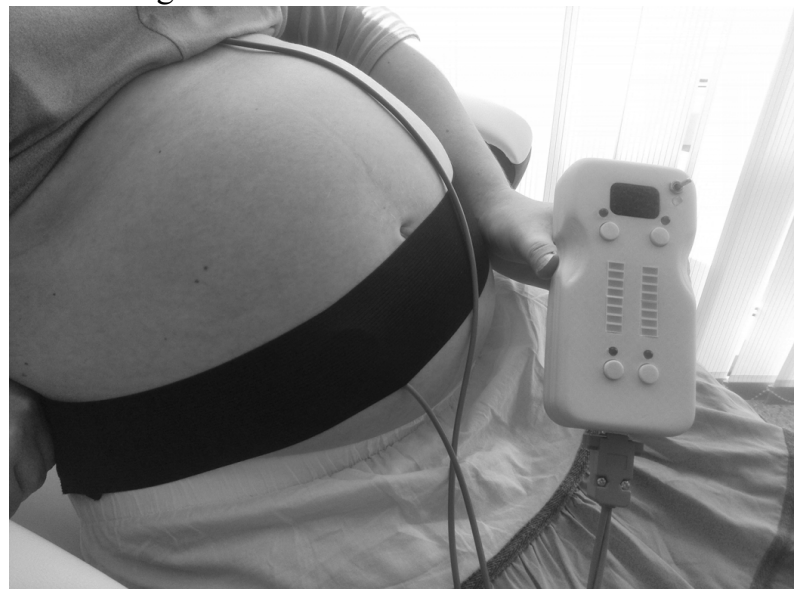

Figure 2. Layout of recorder and piezoceramic transducers adjusted to a pregnant woman's body.

The server of the system is a multiprocessor computer for collection, systematization, gathering, storage, modification, processing, analysis of data obtained from portable recorders. To ensure high-quality connection with translators the server has a fiber-optic communication link with the provider. The server is based on complex programs. They search, identify, process, filter and place the data obtained in a database. All the necessary processing procedures are done automatically. They involves identification and removing artifacts from acoustic signals of heart tones, evaluation of between systolic time intervals, and building of cardiotocograms. 
After evaluating fetal heart rate using cardiotocography, analytical complex of the server begins evaluation of well-being of the fetus. If it is within normal range, a brief conclusion will be sent to a patient's smartphone.

A cardiotocograms, built automatically, is shown in Figure 3; duration 62 minutes.

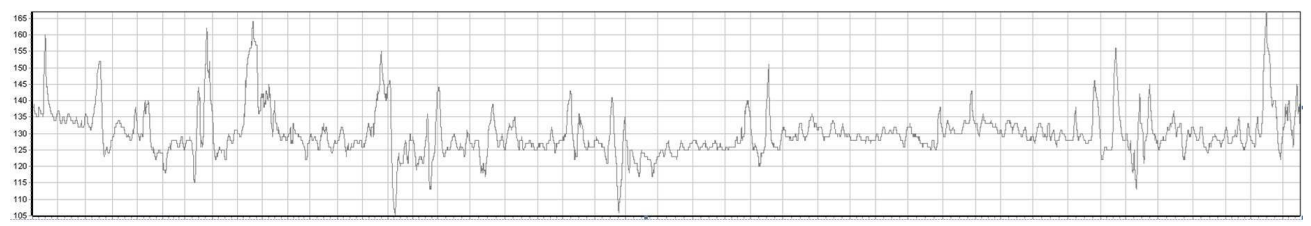

Figure 3. A cardiotocogram, built on the basis of a fetal phonocardiogram.

Comparison of cardiotocograms, built on the basis of a fetal phonocardiogram and built on the basis of an ultrasound investigation, showed imperceptible (no more than 3\%) differences in the assessment of between systolic time intervals.

In case of deviations, anticipated in the settings of the analytical program complex or an abnormal situation, not included in the settings, a relevant entry is registered. indicating a pregnant woman, giving a preliminary conclusion, and coordinating the fragment obtained.

The doctor in charge can track a dynamic pattern of maternal and fetal condition using the doctor's computer work station, view phonocardiogram curves, and analyze calculated assessment parameters.

\section{Results and discussions}

The team of authors has proposed an operation scheme of the mobile system in which the portable units (recorder and the translator) are battery-powered and supports data exchange via a secure wire-free communication Stationary high efficiency elements of the system, the server and the doctor's computer work station are connected by digital links.

Similar in structure and purpose schemes had been proposed previously [6]. But they were intended for use in a hospitals. Digital data were transmitted over a wireless communication channel or through a specialized translator. In the presented device smartphone (serial production) with pre-installed specialized software is used as an intermediary. The proposed operation scheme is less expensive and easier to manufacture. It is not geographically limited due to availability of a mobile communication. Patent applications for the proposed methodology and technical solutions have been filed [7].

The accuracy of recognition algorithms for maternal and fetal heart rates, in comparison with the reference method of ultrasonic cardiotocography, was acceptable: the average error of evaluation ranged from 3 to 6 beats per minute, or less than 3\%. Multiple processing of the same phonocardiogram by a complex of analytical programs gave identical results. That indicates a high stability of the algorithms used.

Compared with fetal electrocardiography of MONICA AN24, recognition algorithms for allocation of heart tones are more effective. Thus, the data processing of a workstation with dual-core Intel Pentium i5 processor with $8 \mathrm{~GB}$ of RAM, was mainly determined by the duration of the original record. The signal duration of 18 minutes was processed and analyzed fully automatically from 25 to 30 seconds and signal duration of 8 hours was processed from 250 to 300 seconds. Such time expenditures of signal processing and analysis are significantly lower than when using fetal electrocardiogram. For example, for processing and analysis of a moderately noisy signal lasting 35 minutes MONICA AN24 needs $60-65$ minutes with an error probability of no more than $15 \%$ [3]. 


\section{Conclusion}

The present study focuses on practical solution to the problem of creating a mobile system for continuous daily monitoring of fetal cardiovascular activity. The developed prototype of a hardware and software system is proceeding to preclinical trials at Tomsk medical institutions. There are all necessary prerequisites for the realization of an individual portable daily monitor. Computing capacities of microcontrollers with low power consumption are enough for solving the above mentioned problems in real-time.

\section{Acknowledgement}

The study was performed within the framework of the Agreement № 14.579.21.0019 (unique identifier for the project - RFMEFI57914X0019) on the «Development of the device for daily monitoring of the fetus and the mother during pregnancy by controlling the parameters of the cardiovascular system based on the acoustic data», signed between the R\&D company «Diagnostika +» LLC and the Ministry of Education and Science of the Russian Federation, and the program of improving competitiveness of the TSU (project No. 8.2.31.2015).

\section{References}

[1] E.N. Zelenko Analysis of amplitude-frequency parameters of heart rate fluctuations in diagnostic testing of fetal distress during pregnancy and childbirth: Ph.D. thesis. (Belarusian State Medical University Press, Minsk,2006)

[2] J. Adam, Rev. Obstet. Gynecol 5(3-4), e132 (2012)

[3] Zubair, Z. Alfirevic, American journal of obstetrics and gynecology 204, 262 (2011) doi: 10.1016/j.ajog.2010.10.686

[4] S. L. Cibull, G. R. Harris, D. M. Nell, J Ultrasound Med 32, 1921 (2013) doi : 10.7863/ultra.32.11.1921

[5] Sh. Bureev, D. S. Zhdanov, I. Ju. Zemlyakov, Method of remote monitoring of physiological parameters of a human body and a mobile system for its implementation Patent, Registration number 2015133653/08 (051 772) (2015)

[6] A. M. Povodator, V. U. Godlevskij, V. L. Stepanov, F. N. Abuzjarov, Ju. V. Naberezhnev, I. V. Isaev, A. D. Zakutskij, Device for monitoring of physiological functions, Patent RU 2200463 C2 (2003)

[7] S. Matyaschuk Home Child: online journal. URL: $\quad$ http://www.domrebenok.ru/blog/bezopasnost-meditsinskogo-ultrazvukaofitsialnoe-zaklyuchenie/?sphrase_id $=3681610$ 